Relations industrielles

Industrial Relations

\title{
Nouveau concepts de conditions de travail
}

\section{James O'Connell-Maher}

Volume 2, numéro 1, 1946

URI : https://id.erudit.org/iderudit/1023678ar

DOI : https://doi.org/10.7202/1023678ar

Aller au sommaire du numéro

Éditeur(s)

Département des relations industrielles de l'Université Laval

ISSN

0034-379X (imprimé)

1703-8138 (numérique)

Découvrir la revue

Citer cet article

O'Connell-Maher, J. (1946). Nouveau concepts de conditions de travail.

Relations industrielles / Industrial Relations, 2(1), 6-6.

https://doi.org/10.7202/1023678ar

Tous droits réservés @ C Département des relations industrielles de l’Université Laval, 1946
Ce document est protégé par la loi sur le droit d'auteur. L'utilisation des services d'Érudit (y compris la reproduction) est assujettie à sa politique d'utilisation que vous pouvez consulter en ligne.

https://apropos.erudit.org/fr/usagers/politique-dutilisation/ 


\section{NOUVEAUX CONCEPTS DE CONDITIONS DE TRAVAIL}

L'histoire de l'humanité nous démontre que les guerres, bien que désastreuses et cruelles, ont cependant toujours contribué, directement ou indirectement, à l'avancement de la civilisation en général ; le conflit mondial qui vient à peine de se terminer ne constitue pas une exception et nous commençons déjà à percevoir ses conséquences dans le domaine des conditions de travail.

L'industrie des peuples démocratiques a, de 1939 à 1945 , été taxée à capacité ; sa tâche était de produire toujours plus et toujours mieux en vue de permettre le triomphe des armées de l'air, de terre et des marines combattantes et marchandes. A cette fin, les chefs d'industries ont demandé à leurs ingénieurs une collaboration des plus étroite en vue d'intensifier la production; les gouvernements ont aussi sollicité et obtenu la coopération nécessaire des travailleurs; organisćs ou non, pour en arriver à cette production optimum.

Il fallait, toutefois, placer les travailleurs dans une situation telle qu'ils soient en mesure de fournir les efforts requis; et l'expérience a prouvé, pendant la guerre, que le seul moyen d'atteindre ce but était d'améliorer les conditions de travail à un niveau qui permettrait ]'effort physique et mental maximum. Les réalisations obtenues sont intéressantes; nous commençons déjà à constater leur intervention comme entité permanente dans cette période de retour à la vie industrielle normale.

L'effort du travailleur à l'usine est d'ordre mental et physique; depuis des annćes, le travail organisé et les gouvernements ont posé les actes nécessaires pour assurer le repos physique de l'employé soit par la limitation de la durée du travail, soit par l'octroi de congés hebdomadaires ou annuels.

Maintenant, tous constatent la nécessité de la tranquillité d'esprit de l'employé : cette détente ne peut s'obtenir que par l'application de nouveaux concepts de sécurité sociale de permanence d'emploi et d'un système toujours amélioré d'orientation professionnelle.

Voici d'ailleurs quelques problèmes qui intéressent actuellement les gouvernants, les industriels et les chefs de mouvements ouvriers ; leur solution ne pourra qu'apporter d'excellents résultats pour l'industrie en général, pour le travailleur, et en définitive, pour la nation :

$1^{\circ}$ Examen médical préalable à l'emploi:-Cette question d'importance vitale sera étudiée par le Bureau international du Travail au cours de sa réunion à Montréal, en septembre et en octobre. Il est inutile de dire que l'adaptation physique à un travail déterminé, surtout au début d'une carrière, est d'importance primordiale non seulement pour l'individu mais aussi pour l'employeur. Ce degré d'adaptation a des ré-. sultats directs sur l'efficacité, la productivité et la durée même de la période de travail dans un emploi déterminé.

$2^{\circ}$ Orientation professionne?le: - L'orientation professionnelle est conditionnée, jusqu'à un certain point, par l'adaptation physique : le système ne sera donc amélioré que si l'examen médical préalable à l'emploi devient un fait accompli. Il n'en demeure pas moins que la détermination de l'aptitude mentale à une tâche précise est extrêmement importante. C'est la raison pour laque!le les éducateurs, les industriels bien pensants et le travail organisé favorisent de plus en plus l'orientation professionnelle non seulement sur le plan familial mais aussi sur le plan scolaire et au cours des études techniques post-scolaires. Pour réussir et progresser dans son travail, il faut y être bien adapté et cette adaptation est impossible si le jeune homme se fie aux circonstances pour déterminer la façon dont il gagnera sa vie.

$\Im^{\circ}$ La permanence d'emploi: - La plupart des gouvernements ont, depuis un certain temps, pris les mesures nécessaires en vue de remédier aux maux du chômage par des systèmes d'assurance ou d'assistance ; c'étaient des remèdes. La nouvelle formule préconisée parle travail organisé, aux Etats-Unis surtout, est la garantie d'un nombre minimum de jours d'emploi, par année, dans l'industrie ; on pourrait qualifier ce système de médecine préventive en matière de chômage.

Le but à atteindre est certainement excellent ; par contre, le tout est conditionné par l'activité économique même de la nation et ce système de permanence d'emploi ne saurait en définitive éliminer ni l'assurance, ni l'assistance chômage.

Le fait pour un employé de savoir qu'on lui garantit du travail pour un temps donné assure donc sa tranquillité d'esprit, en lui garantissant la sécurité.

$4^{\circ}$ La fatigue au travail:- Durant la guerre, des études médicales sérieuses ont été poursuivies en matière de fatigue physique et mentale chez les soldats; récemment, on présentait à l'état-major américain un rapport déterminant d'une façon précise le nombre maximum de jours durant lesquels un soldat pouvait fournir du service actif au combat ou aille.urs. L'industrie - et ce rapport le recommandait - va cer. tainement procéder à des études similaires et en venir à des conclusions médicales précises sur la durée maximum du travail au cours de laquelle l'effort peut être maintenu à son plus haut degré.

On a procédé jusqu'à date par voie de réduction proportionnelle des heures de travail, soit en fonction de l'activité industrielle en géréral, soit en fonction du développement d'une usine en particulier, soit en fonction du marché du travail local ; ce n'est que dans de rares exceptions que la preuve médicale de la durée maximum du travail a été établie. En ce qui concerne les pauses, des standards ont déjà été imposés, mais dans ce cas également, la preuve médicale a manqué.

C'est donc un objectif à atteindre. Plusieurs employeurs dans la Province de Québec sont favorables à ce plan; il ne s'agit que de faciliter la tâche et d'y faire participer toutes les bonnes volontés.

Ces améliorations ne consistent pas en la manifestation d'objectifs toujours supérieurs à atteindre par la classe ouvrière : mais simplement en l'élaboration de systèmes modernes qui permettront au capital de produire davantage en utilisant mieux la main-d'œuvre ; aux travailleurs, de mieux contribuer à la production tout en gagnant plus et en conservant la sécurité qui est chère à tous, et à la nation, d'être assurée d'un meilleur standard de vie et d'une civilisation supérieure.

James O'ConNeil-Maher. 